\title{
Screening, identification, and removal dynamics of a novel iron-manganese removal strain
}

\author{
Wenwei Tang ${ }^{\mathrm{a}, *}$, Lujun $\mathrm{Wu}^{\mathrm{a}}$, Jiemin Gong ${ }^{\mathrm{a}}$, Guoqing $\mathrm{Ye}^{\mathrm{b}}$ and Xinping Zeng ${ }^{\mathrm{b}, *}$ \\ ${ }^{a}$ Department of Chemistry, Tongji University, Shanghai 200092, China \\ ${ }^{b}$ School of Life Sciences and Technology, Tongji University, Shanghai 200092, China
}

\begin{abstract}
In order to investigate biological properties of the dominant strain from the biological activated carbon (BAC) filter column, a novel, dominant iron-and-manganese removal strain, FM-2, was screened from BAC mature biological membrane. By phylogenetic analysis based on 16S rDNA sequence comparison, FM-2 was identified to be Citrobacter freundii. The experimental results indicated that Citrobacter sp. FM-2 could remove $83.6 \%$ Fe (II) and $64.9 \%$ Mn (II) after four days. Inoculum greatly influenced the iron-manganese removal performances. The appropriate inoculum concentration was $5 \%$. The initial concentrations of Fe (II) and Mn (II) had little negative influence on its removal performance. When Fe (II) and Mn (II) concentrations were 32.9 85.7 and $25.7 \sim 46.7 \mathrm{mg} / \mathrm{L}$, respectively, the removal of Fe (II) and Mn (II) followed the first-order dynamical model with the velocity constants of $0.8528 \sim 0.9937 / \mathrm{d}$ and $0.3001 \sim 0.3179 / \mathrm{d}$, respectively.
\end{abstract}

Keywords: Iron-manganese removal strain, Citrobacter $s p$. FM-2, removal efficiency

\section{Introduction}

In recent times, there has been widespread harm of iron and manganese pollution in drinking water sources. Excess amounts of iron will damage the human liver; therefore, areas of high iron contamination often cause an increased incidence of liver disease. Long-term excessive intake of manganese in low doses can cause chronic poisoning, tremors, palsy, mental disorders and permanent disability.

Research and application of iron and manganese removal in micro polluted source water has been developed from air oxidation, contact oxidation to biological fixation and removal technology. There are many families and genera of bacteria which can oxidize iron or manganese and deposit its oxides in cells. They mainly distribute in bacillus, Leptothrix, Sphaerotilus, Pseudomonas and Pedomicrobium, etc. [1,2]. Gheriany et al. [3] found that Leptothrix discophora SS-1 had a promoter action in the oxidation of manganese with iron. $\mathrm{Li}$ [4,5] separated multiple strains, including

\footnotetext{
${ }^{*}$ Corresponding authors: Wenwei Tang, Department of Chemistry, Tongji University, \#1239 Siping Rd, Shanghai 200092, China. Tel./Fax:+86 021 2165983366; E-mail: tangww@tongji.edu.cn.

Xinping Zeng, School of Life Sciences and Technology, Tongji University, \#1239 Siping Rd, Shanghai 200092, China. Tel./Fax:+86 021 2165983366; E-mail: zengxp@tongji.edu.cn.
} 
Gallionella, Leptothrix, Hyphomicrobium and Pseudomonas from biological aerated filter layers that had been operated for four months and greatly affected iron, manganese and arsenic ions. They also found that the oxidation of different strains on pollutants occurred at different depths of the filtering layer.

In the author's previous work, a new manganese-oxidizing strain FM-2 was screened out from the biological activated carbon (BAC) filter column, which was identified as Citrobacter freundii [6]. In this paper, the author separated and screened an efficient strain FM-2, which had a good removal performance of iron and manganese from biological activated carbon (BAC), and conducted the test of identification by $16 \mathrm{~S}$ rDNA sequence and physiological and biochemical experiments. The dynamics of iron and manganese removal in different inoculation quantities and initial concentrations of $\mathrm{Fe}$ (II) and Mn (II) were also analyzed. Results of the research can provide technical support for pollutant treatment of water contaminated by iron and manganese.

\section{Experimental method}

\subsection{Bacteria and medium}

The original bacteria were obtained from mature biofilm in BAC filter column. The main culture media used in this study were JFM medium as described by [7] and PYCM medium [8].

\subsection{Measurement of removal rate of Fe (II) and Mn (II)}

After bacterium was cultivated in PYCM culture media at $27^{\circ} \mathrm{C}$ for an established time, two eppendorf-tubes of PYCM culture media were collected and centrifuged at $12000 \mathrm{rpm}$ for 10 minute. Then $1 \mathrm{~mL}$ of supernatant was collected and diluted with deionizer water to $50 \mathrm{~mL}$. The control sample was PYCM culture media with no bacteria. The concentrations of iron and manganese were measured by phenanthroline spectrophotometry and spectrophotometric oxidation of potassium periodate, respectively [9]. The removal rate of iron or manganese was obtained as follows:

$$
\text { Removal rate }(\%)=(\mathrm{A}-\mathrm{B}) / \mathrm{A} \times 100 \%
$$

where A signifies the iron or manganese concentration in the control group and B is that of the bacteria-treated sample.

\subsection{Isolation and screening of the dominant strain}

Original bacteria were obtained from the mature biofilm in the biological activated carbon filter column, which was used to remove iron and manganese in the experiment. When the mature biological membrane was formed in BAC filter column, $10 \mathrm{~g}$ of active carbon was obtained from the sampling ports and placed in a sterilized conical beaker with a magnetic stir. $100 \mathrm{~mL}$ of sterilized water was added. The conical beaker was stirred for 10 minute and left to stand. Then $2 \mathrm{~mL}$ upper bacteria solution was inoculated into sterilized liquid JFM culture medium for accumulation culture for four days at $27^{\circ} \mathrm{C}$. 
After six concentration gradients $\left(10^{-1}-10^{-6}\right)$ of dilution, one tenth $\mathrm{mL}$ of mixed bacteria solution was collected and coated on a sterilized PYCM solid agar plate. The agar plate was cultivated at $27^{\circ} \mathrm{C}$ for four days. Colonies of different shapes, sizes and colors were examined carefully for isolation. Each type of colony was extracted and streaked across a petri dish until a single strain was obtained. Their iron-manganese removing capacities were examined to select the dominant strain.

\section{4. $16 S$ rDNA sequence alignment and phylogeny analysis}

DNA was extracted from cultures using the SK1201-UNIQ-10 DNA extraction kit (Sangon Biotech Co., Ltd., Shanghai, China). The 16S rDNA gene was amplified using the primers 27F and 1492R [10] in a standard 35-cycle PCR with an annealing temperature of $55^{\circ} \mathrm{C}$. The cloned PCR products were sequenced by Sangon Biotech Co., Ltd. Then $16 \mathrm{~S}$ rDNA gene sequences were aligned manually using the nucleotide blast program. CLUSTAL W was used to carry out the sequence homology analysis .A Phylogenetic tree was generated by neighbor-joining using Mega 4.0. Bootstrap analysis was used to estimate the reliability of phylogenetic reconstructions using 1000 replicates.

\section{Results and discussions}

\subsection{Separation and screening of the dominant bacteria}

Eight bacterial strains were separated and purified from the bacterial mixture, in accordance with their shape, size, color, edge and other characteristics. Figure 1 displays the $\mathrm{Fe}$ (II) and $\mathrm{Mn}$ (II) removal capabilities of different bacteria. One can observe that the bacteria of item 2, item 5 and item 6 exhibit favorable iron removal performances, with four days removal ratios higher than $80 \%$, while the bacteria of item 2 exhibit a remarkable manganese removal capability when compared with the removal capability of the others. Accordingly, the bacteria of item 2 was selected as the object in the present study and named FM-2. The 4d Fe (II) and Mn (II) removal ratios of FM-2 can be up to $83.6 \%$ and $64.9 \%$, respectively.

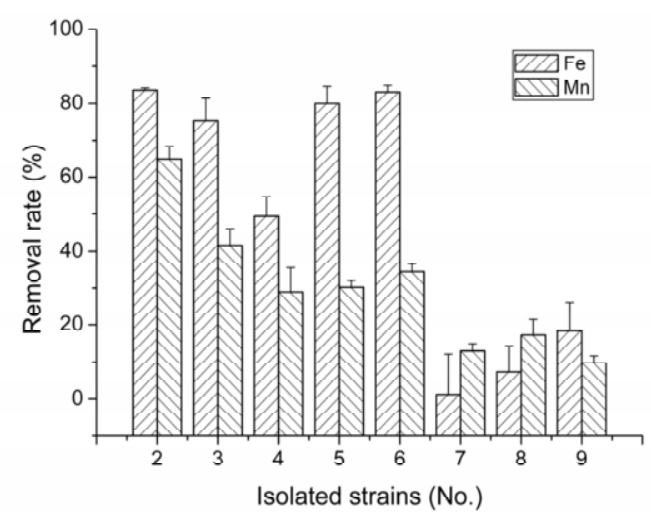

Fig. 1. Fe (II) and Mn (II) removal effect of diferent isolated strains. 


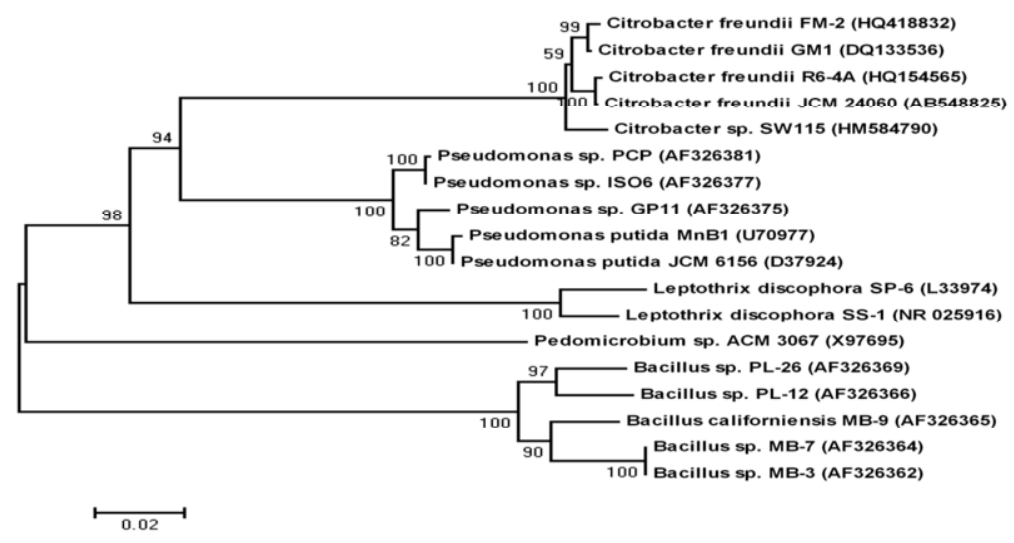

Fig. 2. 16S rDNA phylogenetic tree of Citrobacter sp. FM-2.

Accession numbers are given in brackets. Numbers near the branches are bootstrap probability values from 1000 replicates. Scale bar represents $2 \%$ difference in nucleotide.

\section{2. $16 \mathrm{~S}$ rDNA sequence alignment and phylogeny analysis}

A 16S rDNA sequence of $1501 \mathrm{bp}$ was obtained from Strain FM-2. The sequence alignment in GenBank showed that 16S rDNA sequence of FM-2 was closely related to that of many Citrobacters (99\% identical to Citrobacter freundii GM1).

A phylogenetic tree based on 16S rDNA genes, which were obtained from these Citrobacters and other iron-manganese oxidizing strains, revealed the presence of several distinct phylogenetic clusters (Figure 2). The oxidizing strains in Bacillus, Pseudomonas, Leptothrix or Pedomicrobium formed separate phylogenetic clusters. Strain FM-2 fell within the Citrobacter freundii cluster, which was consistent with the results from BLAST. The accession name and number from GenBank were Citrobacter sp. FM-2 and HQ418832.

\subsection{Dynamical models of Fe (II) and Mn (II) removal by FM-2}

\subsubsection{Effects of inoculum concentration on the dynamic models of Fe (II) and Mn (II) removal}

The experimental results in Figures 3(A) and 3(B) indicate that the effects of inoculum concentrations on both $\mathrm{Fe}$ (II) and Mn (II) removal performances are similar. With the increase of inoculum concentrations, the lag phase of bacteria growth is shortened. The period to achieve the same removal results is reduced as well. These variations are more distinct in the early stages. Additionally, when the inoculum concentration reaches and exceeds 5\%, the removal effects present no obvious advantages, especially at the later stage after three days. The analyses suggest that the amount of bacteria is small in culture medium at the early stage, and thus, the Fe (II) and Mn (II) removal ratios increase significantly with the increase of inoculum concentration. Furthermore, after culturing for a certain period, the source of nutrients in the culture solution tends to be the constraint in bacterial multiplication. The increasing amount of inoculum cannot generate the corresponding enhancement of removal ratios. Considering both the reduction of bacterial culture period and the removal ratios comprehensively, a 5\% inoculum concentration was suitable.

Linear regressions were performed on obtained data, with the coordinate axes being $\ln \left(\mathrm{C}_{0} / \mathrm{C}\right)$ and $t$, respectively. The results are shown in Figures 4(A) and 4(B). Subsequently, significance tests were carried out on the correlation coefficients of the equations. Y.Y. Li and C.R. Hu [11] indicated that a 
close, linear relationship exists only when $|\mathrm{R}|>\mathrm{R}_{\min }$, at the confidence levels of $95 \%$ or $99 \%(\alpha=0.05$ or $\alpha=0.01$ ). As listed in Table 1 , the dynamical equations satisfy the relationship $|R|>R_{\min }$; therefore, the Fe (II) and Mn (II) removal can be expressed by the first-order dynamical model.
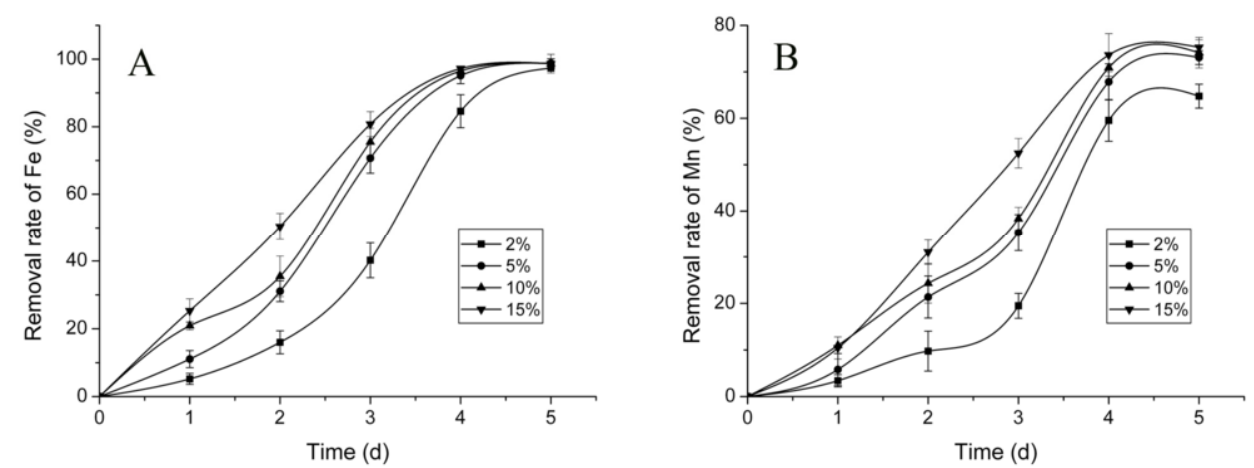

Fig. 3. Effects of inoculum concentration on removal effect: (A) Initial $\mathrm{Fe}^{2+}$ concentration was $98.4 \mathrm{mg} / \mathrm{L}$; (B) Initial $\mathrm{Mn}{ }^{2+}$ concentration was $43.2 \mathrm{mg} / \mathrm{L}$.
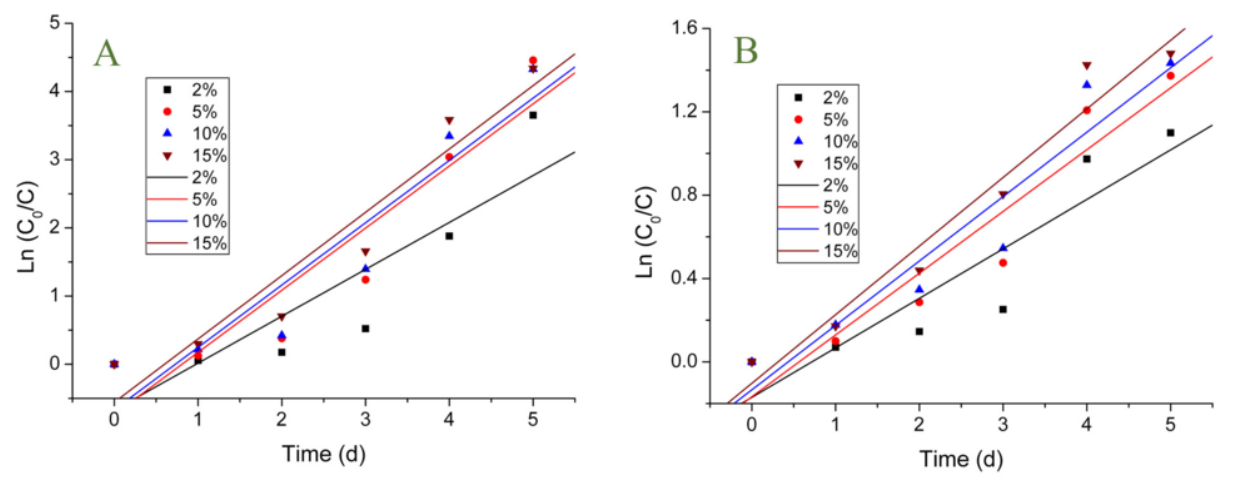

Fig. 4. Effects of inoculum concentration on the dynamic models of: (A) the iron removal; (B) the manganese removal.

Table 1

Reaction kinetics equation of $\mathrm{Fe}^{2+}$ and $\mathrm{Mn}^{2+}$ removal with different inoculums, respectively

\begin{tabular}{lllccc}
\hline \multirow{2}{*}{ Object } & \multirow{2}{*}{ Inoculum (\%) } & Reaction kinetics equation & \multicolumn{3}{c}{ The test of correlation coefficient $\mathrm{R}$} \\
\cline { 4 - 6 } & & $\mathrm{Ln}\left(\mathrm{C}_{0} / \mathrm{C}\right)=0.6881 \mathrm{t}-0.6724$ & $\alpha=0.01 / 0.05$ & Significant \\
\hline \multirow{3}{*}{$\mathrm{Fe}^{2+}$} & 5 & $\operatorname{Ln}\left(\mathrm{C}_{0} / \mathrm{C}\right)=0.9111 \mathrm{t}-0.7372$ & 0.9843 & 0.8114 & $*$ \\
& 10 & $\operatorname{Ln}\left(\mathrm{C}_{0} / \mathrm{C}\right)=0.9141 \mathrm{t}-0.6669$ & 0.9447 & 0.9172 & $* *$ \\
& 15 & $\operatorname{Ln}\left(\mathrm{C}_{0} / \mathrm{C}\right)=0.9290 \mathrm{t}-0.5584$ & 0.9612 & 0.9172 & $* *$ \\
$\mathrm{Mn}^{2+}$ & 2 & $\operatorname{Ln}\left(\mathrm{C}_{0} / \mathrm{C}\right)=0.2376 \mathrm{t}-0.1705$ & 0.9185 & 0.9172 & $* *$ \\
& 5 & $\operatorname{Ln}\left(\mathrm{C}_{0} / \mathrm{C}\right)=0.2966 \mathrm{t}-0.1678$ & 0.9550 & 0.9172 & $* *$ \\
& 10 & $\operatorname{Ln}\left(\mathrm{C}_{0} / \mathrm{C}\right)=0.3092 \mathrm{t}-0.1345$ & 0.9579 & 0.9172 & $* *$ \\
& 15 & $\operatorname{Ln}\left(\mathrm{C}_{0} / \mathrm{C}\right)=0.3294 \mathrm{t}-0.1032$ & 0.9791 & 0.9172 & $* *$ \\
\hline
\end{tabular}

Note: ** Highly significant, * significant, the same as in Table 2. 
As displayed in the dynamical equations, the iron removal rate increases rapidly with the increase of inoculum concentration. The removal rate increases by $35.0 \%$ as the inoculum concentration increases from $2 \%$ to $15 \%$. When the inoculum concentration is increased from $2 \%$ to $5 \%$, the removal rate increases correspondingly from $2 \%$ to $32.4 \%$, while the inoculum concentrations are $10 \%$ and $15 \%$, compared with the results from a 5\% inoculum concentration, the iron removal rate only increases by $0.3 \%$ and $2.0 \%$, respectively, mainly due to the constraints from nutrition substance. The manganese removal displays similar tendencies to that of the iron removal. The removal rate increases by $38.6 \%$ as the inoculum increases from $2 \%$ to $15 \%$. The most rapid variation also appears when the inoculum is increased from $2 \%$ to $5 \%$, with the rate increasing from $2 \%$ to $19.9 \%$.

\subsubsection{Removal of dynamical models under different concentrations of Fe (II) and Mn (II)}

The Fe (II) and Mn (II) concentrations were measured at different times and presented in Figures 5(A) and 5(B), respectively. Linear regressions were then performed, in the form of $\ln \left(\mathrm{C}_{0} / \mathrm{C}\right)$ with the variation of time $t$, as displayed in Figures 6(A) and 6(B). The results indicate a favorable linear relationship between $\ln \left(\mathrm{C}_{0} / \mathrm{C}\right)$ and $t$.

Significance tests were carried out on the correlation coefficients of the equations. As shown in Table 2, $|\mathrm{R}|$ in the models is greater than $\mathrm{R}_{\min }$. Therefore, the Fe (II) and Mn (II) removal behaviors can be descried by the first-order dynamical model. Under a temperature of $27^{\circ} \mathrm{C}$ and a $\mathrm{pH}$ level of 7.0 ,
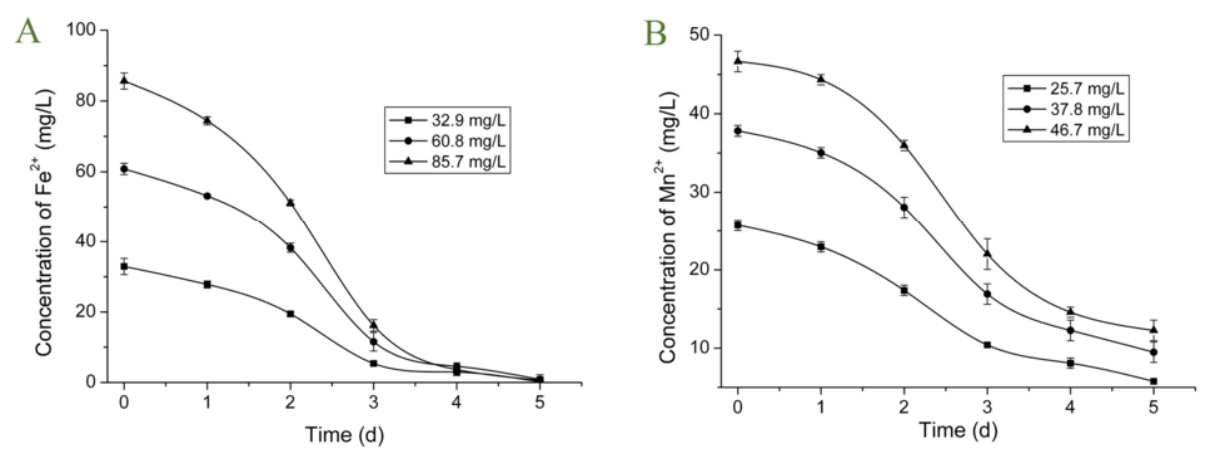

Fig. 5. Removal effect with different initial concentrations by FM-2: (A) Initial $\mathrm{Fe}^{2+}$ concentration was $32.9,60.8$ and $42.8 \mathrm{mg} / \mathrm{L}$, respectively; (B) Initial $\mathrm{Mn}^{2+}$ concentration was $46.7,37.8$ and $25.7 \mathrm{mg} / \mathrm{L}$, respectively.
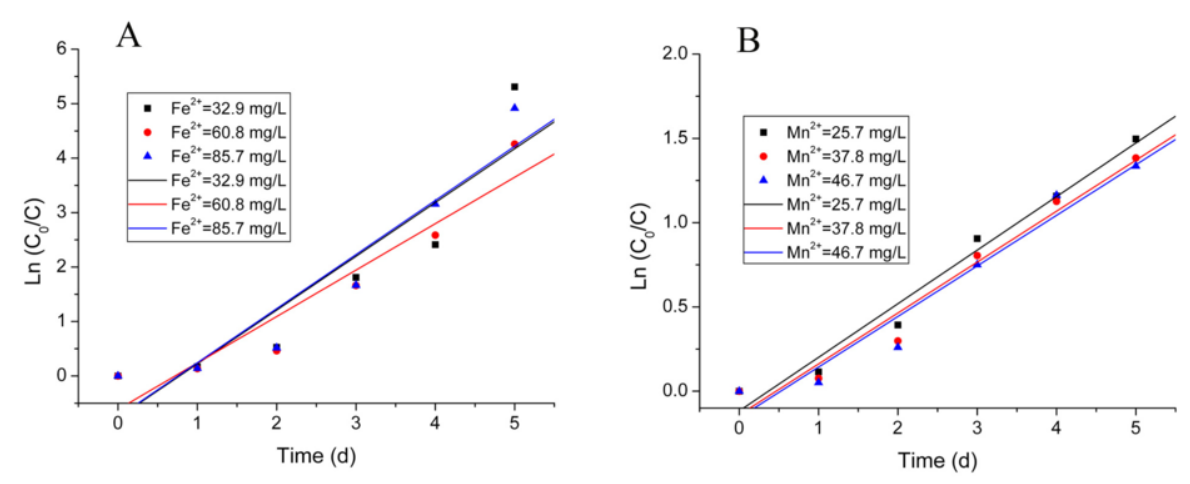

Fig. 6. Diagram of $\mathrm{Ln}\left(\mathrm{C}_{0} / \mathrm{C}\right)$-t. (A) at different initial $\mathrm{Fe}^{2+}$ concentrations; (B) at different initial $\mathrm{Mn}^{2+}$ concentrations. 
Table 2

Reaction kinetics equation of $\mathrm{Fe}^{2+}$ and $\mathrm{Mn}^{2+}$ removal by $\mathrm{FM}-2$, respectively

\begin{tabular}{cccccc}
\hline \multirow{2}{*}{ Object } & \multirow{2}{*}{$\begin{array}{l}\text { Initial } \\
\text { concentration } \\
(\mathrm{mg} / \mathrm{L})\end{array}$} & Reaction kinetics equation & $|\mathrm{R}|$ & $\alpha=0.01$ & Significant \\
\cline { 4 - 6 } & 32.9 & $\mathrm{Ln}\left(\mathrm{C}_{0} / \mathrm{C}\right)=0.9876 \mathrm{t}-0.7644$ & 0.91999 & 0.9172 & $* *$ \\
\hline \multirow{2}{*}{$\mathrm{Fe}^{2+}$} & 60.8 & $\mathrm{Ln}\left(\mathrm{C}_{0} / \mathrm{C}\right)=0.8528 \mathrm{t}-0.6146$ & 0.95328 & 0.9172 & $* *$ \\
& 85.7 & $\mathrm{Ln}\left(\mathrm{C}_{0} / \mathrm{C}\right)=0.9937 \mathrm{t}-0.7517$ & 0.94903 & 0.9172 & $* *$ \\
\hline \multirow{3}{*}{$\mathrm{Mn}^{2+}$} & 25.7 & $\mathrm{Ln}\left(\mathrm{C}_{0} / \mathrm{C}\right)=0.3179 \mathrm{t}-0.1168$ & 0.98825 & 0.9172 & $* *$ \\
& 37.8 & $\mathrm{Ln}\left(\mathrm{C}_{0} / \mathrm{C}\right)=0.3021 \mathrm{t}-0.1403$ & 0.98191 & 0.9172 & $* *$ \\
& 46.7 & $\mathrm{Ln}\left(\mathrm{C}_{0} / \mathrm{C}\right)=0.3001 \mathrm{t}-0.1569$ & 0.97529 & 0.9172 & $* *$ \\
\hline
\end{tabular}

the $\mathrm{Fe}$ (II) removal rate of FM-2 is approximately $0.8528 \sim 0.9937$ per day when the initial $\mathrm{Fe}$ (II) concentration is approximately $32.9 \sim 85.7 \mathrm{mg} / \mathrm{l}$, while the Mn (II) removal rate is approximately $0.3001 \sim 0.3179$ per day when the initial Mn (II) concentration is approximately $25.7 \sim 46.7 \mathrm{mg} / \mathrm{L}$. In conclusion, the $\mathrm{Fe}$ (II) removal rate far exceeds the Mn (II) removal rate, indicating a stronger removal capability. Moreover, the removal rates of $\mathrm{Fe}$ (II) and Mn (II) are not significantly affected by the variation of initial concentration.

\section{Conclusion}

After being separated and purified, eight bacterial strains with $\mathrm{Fe}$ (II) and $\mathrm{Mn}$ (II) removal capabilities were obtained, in which FM-2 displayed the most favorable removal performances. The $\mathrm{Fe}$ (II) and Mn (II) removal ratios after four days can be up to $83.6 \%$ and $64.9 \%$, respectively. FM-2 was identified to be Citrobacter freundii. As the inoculum concentration increased from $2 \%$ to $15 \%$, the Fe (II) and Mn (II) removal rates increased by $35.0 \%$ and $38.6 \%$, respectively. Particularly, when the inoculum concentration is increased from $2 \%$ to $5 \%$, the variation of removal rates is most significance. The dynamical processes of Fe (II) and Mn (II) removal can be described by the first-order dynamical model. When Fe (II) and Mn (II) concentrations were 32.9 85.7 and 25.7 46.7 $\mathrm{mg} / \mathrm{L}$, respectively, the removal of Fe (II) and Mn (II) followed the first-order dynamical model with the velocity constants of $0.8528 \sim 0.9937 / \mathrm{d}$ and $0.3001 \sim 0.3179 / \mathrm{d}$, respectively.

\section{Acknowledgement}

This work was supported by the National Natural Science Foundation of China (No. 21277098), the Natural Science Foundation of Shanghai (No. 10ZR1432500), and the Shanghai Municipal Commission of Economy and Informatization (No. 12CH-12).

\section{References}

[1] S.Y. Qin, F. Ma and P. Huang, Ferrous-manganese oxidizing bacteria from the nature water, Environmental Science 29 (2008), 1649-1654.

[2] S.Y. Qin, F. Ma and P. Huang, Fe (II) and Mn (II) removal from drilled well water: A case study from a biological treatment unit in Harbin, Desalination 245 (2009), 183-193. 
[3] E.L Gheriany, D. Bocioaga and A.G. Hay, Iron requirement for Mn (II) oxidation by leptothrix discophora SS-1,Applied and Environmental Microbiology 75 (2009), 1229-1235.

[4] X.K. Li, Z.R. Chu, Y.J. Liu, M.T. Zhu, Y. Liu and J. Zhang, Molecular characterization of microbial populations in full-scale biofilters treating iron, manganese and ammonia containing groundwater in Harbin, China, Bioresource Technology 147 (2013), 234-239.

[5] Y. Liu, X.K. Li, Z.R. Chu, Y.H. Ren and J. Zhang, Distribution and genetic diversity of the microorganisms in the biofilter for the simultaneous removal of arsenic, iron and manganese from simulated groundwater, Bioresource Technology 1 (2014), 1-5.

[6] W.W. Tang, J. Xia, X.P. Zeng, L.J. Wu and G.Q. Ye, Biological characteristics and oxidation mechanism of a new manganese-oxidizing bacteria FM-2, Bio-Medical Materials and Engineering 24 (2014), 703-709.

[7] S.F. Bilinski, H. Bilinski, R. Grbac, J. Zunic, M. Necemer and D. Hanzel, Multidisciplinary work on barium contamination of the karstic upper kupa river drainage basin (croatia and slovenia); calling for watershed management, Environmental Geochemistry and Health 29 (2007), 69-79.

[8] J.C. Xu, G. Chen, X.F. Huang, G.M. Li, J. Liu, N. Yang and S.N. Gao, Iron and manganese removal by using manganese ore constructed wetlands in the reclamation of steel wastewater, Journal of Hazardous Materials 169 (2009), 309-317.

[9] Ministry of Environmental Protection of the People's Republic of China, the Manual to Monitor and Analysis Water and Wastewater, 4th ed., Environmental Science Press, Beijing, China, 2002.

[10] W.G. Weisburg, S.M. Barns, D.A. Pelletier and D.J. Lane, 16S ribosomal DNA amplification for phylogenetic study, Bacteriol 173 (2) 1991, 697-703.

[11] Y.Y. Li and C.R. Hu, Experiment Design and Date Processing, Chemical Industry Press, Beijing, 2003. 